HD-TVP-98-04

\title{
Flow Equations for the Hénon-Heiles Hamiltonian
}

\author{
Daniel Cremers" and Andreas Mielkef \\ Institut für Theoretische Physik, \\ Ruprecht-Karls-Universität, \\ Philosophenweg 19, \\ D-69120 Heidelberg, F.R. Germany
}

November 14, 2016

\begin{abstract}
The Hénon-Heiles Hamiltonian was introduced in 1964 [1] as a mathematical model to describe the chaotic motion of stars in a galaxy. By canonically transforming the classical Hamiltonian to a Birkhoff-Gustavson normalform Delos and Swimm obtained a discrete quantum mechanical energy spectrum. The aim of the present work is to first quantize the classical Hamiltonian and to then diagonalize it using different variants of flow equations, a method of continuous unitary transformations introduced by Wegner in 1994 [2]. The results of the diagonalization via flow equations are comparable to those obtained by the classical transformation. In the case of commensurate frequencies the transformation turns out to be less lengthy. In addition, the dynamics of the quantum mechanical system are analyzed on the basis of the transformed observables.

PACS-numbers: $03.65 .-\mathrm{w}$ (Quantum mechanics),

05.45. $+\mathrm{b}$ (Theory and models of chaotic systems)
\end{abstract}

Keywords: Hénon-Heiles Hamiltonian, Quantum chaos, Flow equations

\section{Introduction}

The Hénon-Heiles Hamiltonian describes two one-dimensional harmonic oscillators with a cubic interaction. It is one of the simplest Hamiltonians to display soft chaos in classical mechanics: by increasing the total energy a transition from an integrable to an ergodic system is induced. Originally conceived to model the chaotic motion of stars in a galaxy it later became an important milestone in the development of the theory of chaos [3], partly because of the conceptual simplicity of the model.

In order to investigate this continuous loss of integrability with growing total energy Gustavson [4] transformed the classical Hamiltonian by a series of canonical transformations to a Birkhoff-Gustavson normal form [5], which allowed him to construct an additional constant of motion. Thus he was able to analytically reproduce Poincaré surfaces of section as obtained by numerical integration.

The present work is based upon two publications ([6] and [7]) in which Delos and Swimm used the classical Birkhoff transformation [5] as applied by Gustavson to analyze how the classically chaotic

\footnotetext{
${ }^{1}$ E-mail: cremers@itb.biologie.hu-berlin.de ,new permanent address: Innovationskolleg Theoretische Biologie, Humbold Universität Berlin, Invalidenstraße 43, D-10115 Berlin

${ }^{2}$ E-mail: mielke@tphys.uni-heidelberg.de
} 
behavior of the system is transformed into quantum mechanics. The Birkhoff-Gustavson normal form is a power series in oscillator Hamiltonians and thus allows a direct determination of a quantum mechanical eigenvalue spectrum from the classical Hamiltonian. For a fixed set of parameters Delos and Swimm analytically calculated the spectrum of the Hénon-Heiles Hamiltonian which reproduced the eigenvalues obtained by numerical diagonalization of finite matrices.

The main problem of the quantum mechanical Hénon-Heiles Hamiltonian is the fact that it is not bounded from below. It contains a cubic potential. The classical motion discussed by Gustavson and by Delos and Swimm corresponds to initial conditions near the local minimum of the potential and to an energy that is below the saddle point value of the potential. By these conditions the classical motion is always restricted to a finite region. In the corresponding quantum problem, the particle will always tunnel through the barrier. Therefore the eigenvalue spectrum calculated by Delos and Swimm is not the real eigenvalue spectrum of the Hamiltonian. It describes effective states that can be used to describe the dynamics near the minimum of the potential and for times that are small compared to the escape time.

The aim of the present paper is to first quantize the classical Hamiltonian and to then diagonalize it using the method of flow equations which was introduced by Wegner [2] in 1994. It is clear that concerning the tunneling problem, the flow equations have the same limitation as the quantization of the Birkhoff-Gustavson normal form by Delos and Swimm. The bound states and the eigenvalues obtained using flow equations allow only an effective description for small times. One advantage of the flow equations is that a correct and simple treatment of the system is possible even if the two frequencies of the harmonic oscillators are commensurate. This is not the case if Birkhoff-Gustavson normal form is quantized. Furthermore, the quantum mechanical treatment allows a description of the dynamics.

The structure of the paper is as follows. The next section offers a general introduction to the flow equation method. In sections 3 and $\emptyset$ their application in two variants to the Hénon-Heiles model is treated. Section 5 contains some results of the diagonalization: in a table the energy eigenvalues obtained in the flow equation approach are compared with those obtained by numerical matrix diagonalization for a fixed coupling constant. A graph shows the dependence of the calculated eigenvalues upon the coupling strength. In section 6 a case of commensurate frequencies is treated and a similar table of eigenvalues is obtained. Section 7 offers a method to investigate the dynamics of the quantum mechanical system, transition amplitudes between the eigenstates of the uncoupled system are determined. The effect of growing coupling strength upon the transition amplitudes is shown. The last section contains a summary of the results of the present work, gives a comparison to the work of Delos and Swimm and a discussion of the limitations of the Birkhoff-Gustavson transformation.

\section{Flow Equations}

The method of flow equations consists in a continuous unitary transformation of a given Hamilton operator $H$, which can be written in differential form:

$\frac{d H(\ell)}{d \ell}=[\eta(\ell), H(\ell)]$.

There are several possibilities to choose the antihermitean generator $\eta$ so that $H(\infty)$ becomes diagonal. Wegner [2] proposed:

$\eta(\ell)=\left[H_{d}(\ell), H(\ell)\right]=\left[H_{d}(\ell), H_{r}(\ell)\right]$,

where $H_{d}$ and $H_{r}$ are the diagonal and the off-diagonal portions of the Hamilton operator respectively. A detailed argument for the usefulness of this choice of the generator can be found in [2]. But the consistency can be easily verified, since in the limit $\ell \rightarrow \infty$ as $H(\ell)$ becomes more diagonal $\eta(\ell)$ will vanish and so will $\frac{d H(\ell)}{d \ell}$. 
The flow equation method has been applied to various models (see e.g. [8, 9, 10, 11, 12]). The general behavior is such that terms that appear in $\eta(2.2)$ will result in new terms in the transformed Hamilton operator by (2.1). If this iterative process does not result in a closed set of differential equations it can be forced into such by defining an order for the appearing terms - e.g. the number of creation operators in them - and neglecting all terms of higher order. This approach will be called cut-off.

A second approach to handle the system of differential equations (2.1) and (2.2) for a given Hamilton operator $H=H_{d}(0)+\lambda H_{r}(0)$ is to define the transformed Hamilton operator as a power series in the coupling constant $\lambda$

$H(\ell)=\sum_{k=0}^{\infty} \lambda^{k} H_{k}(\ell)$.

The coefficients $H_{k}(\ell)$ can be determined iteratively and this method shall be called iteration. Both of these procedures - the cut-off and the iteration - will become more transparent as they are applied to the Hénon-Heiles Hamiltonian in the next two sections.

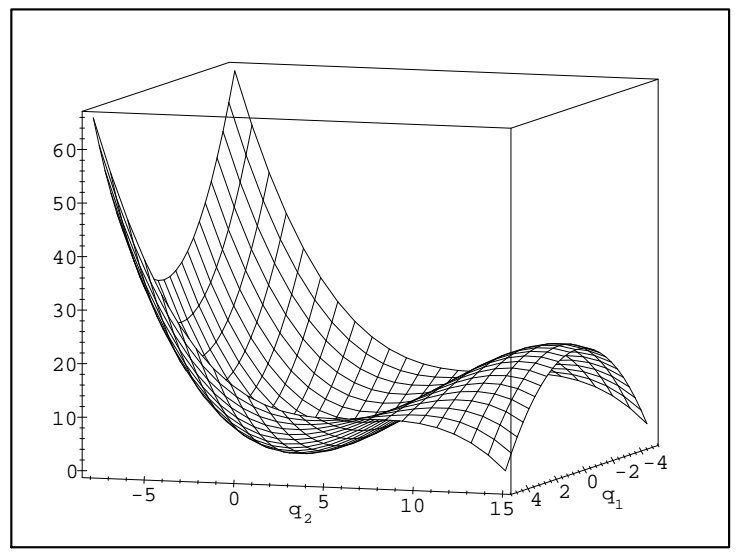

Figure 1: Hénon-Heiles potential (3.1)

$(\lambda=-0.1, n=0.1, w=1.3, v=0.7)$

\section{The Cut-off Procedure}

The Hénon-Heiles Hamiltonian can be expressed as a function of two spatial coordinates $q_{1}$ and $q_{2}$ and the two momenta $p_{1}$ and $p_{2}$ (see figure 1):

$H=\frac{1}{2} w\left(p_{1}^{2}+q_{1}^{2}\right)+\frac{1}{2} v\left(p_{2}^{2}+q_{2}^{2}\right)+\lambda q_{2}\left(q_{1}^{2}+n q_{2}^{2}\right)$.

Quantizing this classical Hamiltonian using the operators

$a:=\frac{1}{\sqrt{2}}(\hat{q}+i \hat{p}), \quad a^{\dagger}:=\frac{1}{\sqrt{2}}(\hat{q}-i \hat{p})$.

will give the Hamilton operator

$H=w a^{\dagger} a+v b^{\dagger} b+\lambda\left(b^{\dagger}+b\right)\left(\left(a^{\dagger}+a\right)^{2}+n\left(b^{\dagger}+b\right)^{2}\right)$,

where the coupling constant $\lambda$ has been rescaled and the constant term dropped. 
This Hamilton operator is to be transformed into the quantum mechanical equivalent of a Birkhoff normalform, a power series in oscillator Hamiltonians:

$H \longrightarrow \sum_{k, m=0}^{\infty} \beta_{k m}\left(a^{\dagger} a\right)^{k}\left(b^{\dagger} b\right)^{m}=\sum_{k, m=0}^{\infty} w_{k m} a^{\dagger k} a^{k} b^{\dagger m} b^{m}$.

The generalized frequencies $w_{k m}$ are obtained from the transformed Hamilton operator $H(\ell)$ in the limit $l \rightarrow \infty$. A consistent ansatz is $H(\ell)=H_{d}(\ell)+H_{r}(\ell)$ where:

$$
\begin{aligned}
H_{d}(\ell)= & w(\ell) a^{\dagger} a+v(\ell) b^{\dagger} b \\
+ & \lambda^{2}\left[w_{00}(\ell)+w_{20}(\ell) a^{\dagger 2} a^{2}+w_{02}(\ell) b^{\dagger 2} b^{2}+w_{11}(\ell) a^{\dagger} a b^{\dagger} b\right] \text { and } \\
H_{r}(\ell)=\lambda & {\left[\left(a^{\dagger 2}+a^{2}\right)\left(b^{\dagger}+b\right) x_{1}(\ell)+\left(a^{\dagger 2}-a^{2}\right)\left(b^{\dagger}-b\right) x_{2}(\ell)\right.} \\
& +\left(a^{\dagger} a\right)\left(b^{\dagger}+b\right) x_{3}(\ell)+\left(b^{\dagger 3}+b^{3}\right) x_{4}(\ell) \\
& \left.+\left(b^{\dagger 2} b+b^{\dagger} b^{2}\right) x_{5}(\ell)+\left(b^{\dagger}+b\right) x_{6}(\ell)\right]
\end{aligned}
$$

The $\ell$-dependent coefficients are determined from (2.1) and (2.2) which combine to:

$\frac{d H}{d \ell}=\left[\left[H_{d}, H_{r}\right], H_{d}\right]+\left[\left[H_{d}, H_{r}\right], H_{r}\right]$.

Neglecting all terms of third order in $\lambda$ or higher we obtain the following set of differential equations by coefficient matching:

$$
\begin{aligned}
w_{00}^{\prime} & =-4 x_{1}^{2} v-8 x_{1}^{2} w-8 x_{1} x_{2} v-16 x_{1} x_{2} w-36 x_{4}^{2} v-2 x_{6}^{2} v-4 x_{2}^{2} v-8 x_{2}^{2} w \\
w^{\prime} & =-8 x_{1}^{2} v-16 x_{1}^{2} w-16 x_{1} x_{2} v-32 x_{1} x_{2} w-2 x_{3}^{2} v-4 x_{3} x_{6} v-8 x_{2}^{2} v-16 x_{2}^{2} w \\
v^{\prime} & =-16 x_{1}^{2} w-16 x_{1} x_{2} v-108 x_{4}^{2} v-4 x_{5}^{2} v-8 x_{5} x_{6} v-16 x_{2}^{2} w \\
w_{20}^{\prime} & =-4 x_{1}^{2} v-16 x_{1} x_{2} w-2 x_{3}^{2} v-4 x_{2}^{2} v \\
w_{11}^{\prime} & =-32 x_{1}^{2} w-32 x_{1} x_{2} v-8 x_{3} x_{5} v-32 x_{2}^{2} w \\
w_{02}^{\prime} & =-54 x_{4}^{2} v-6 x_{5}^{2} v \\
x_{1}^{\prime} & =-x_{1} v^{2}-4 x_{1} w^{2}-4 x_{2} v w \\
x_{2}^{\prime} & =-4 x_{1} v w-x_{2} v^{2}-4 x_{2} w^{2} \\
x_{3}^{\prime} & =-x_{3} v^{2} \\
x_{4}^{\prime} & =-9 x_{4} v^{2} \\
x_{5}^{\prime} & =-x_{5} v^{2} \\
x_{6}^{\prime} & =-x_{6} v^{2}
\end{aligned}
$$

The equations for $x_{3}, \ldots, x_{6}$ are all of similar type, the equations for $w_{i j}$ are uncoupled. The system can therefore be reduced to five equations. It could not be solved analytically. But the asymptotic behavior for large $\ell$ is the following:

Assume that $w(\ell) \approx w(\infty)=w_{\infty}$ and $v(\ell) \approx v(\infty)=v_{\infty}$. Then the off-diagonal elements show an exponential decay (except in the case of commensurate frequencies $2 w_{\infty}+v_{\infty}=0$ ):

$$
\begin{aligned}
& x_{1}(\ell)=c_{0} \exp \left(-\left(2 w_{\infty}+v_{\infty}\right)^{2} \cdot \ell\right) \\
& x_{2}(\ell)=c_{0} \exp \left(-\left(2 w_{\infty}+v_{\infty}\right)^{2} \cdot \ell\right) \\
& x_{3}(\ell)=d_{0} \exp \left(-v_{\infty}^{2} \cdot \ell\right) .
\end{aligned}
$$


The diagonalized Hamilton operator was determined by numerical integration of (3.7) using a RungeKutta procedure. For various sets of fixed parameters the approximation was improved by extending the calculation to all terms up to fourth order in $\lambda$. The resulting set of differential equations for coefficients $x_{1}, \ldots, x_{48}, w, v$ and the coefficients $w_{i j}$ corresponding to the diagonal operators $\left(a^{\dagger i} a^{i}\right)\left(b^{\dagger j} b^{j}\right)$ were also determined by numerical integration. An eigenvalue spectrum obtained from the transformed Hamiltonian in the limit $\ell \rightarrow \infty$ was calculated for different values of the coupling constant $\lambda$. Results of these calculations are presented in section 5 .

\section{The Iterative Procedure}

A far more elegant way to solve the flow equation (2.1) for the Hénon-Heiles Hamiltonian is an iterative calculation of the $H_{k}(\ell)$ defined in (2.3). It avoids the numerical integration applied in the previous section and allows a better insight into the transformation mechanism, specifically the behavior in the case of commensurate frequencies.

The Hamiltonian (3.3) is given in the form

$H(0)=H_{0}(0)+\lambda H_{1}(0) \quad$ with $\quad H_{0}(0)=w a^{\dagger} a+v b^{\dagger} b$.

The transformed Hamiltonian is defined as a power series in $\lambda(2.3)$. In deviation from the original choice (2.2) the generator is now defined as:

$\eta(\ell):=\left[H_{0}, H(\ell)\right]=\sum_{k=1}^{\infty} \lambda^{k} \eta_{k}(\ell), \quad$ where $\quad \eta_{k}=\left[H_{0}, H_{k}\right]$.

This choice of $\eta$ is in accordance with the classical Birkhoff transformation as applied by Gustavson [4]. It makes the iterative calculation simpler, since commutation with $H_{0}$ will reproduce a given operator term $a^{\dagger k} a^{r} b^{\dagger m} b^{n}$ :

$\left[\left[H_{0}, a^{\dagger k} a^{r} b^{\dagger m} b^{n}\right], H_{0}\right]=-\varepsilon_{k r m n} a^{\dagger k} a^{r} b^{\dagger m} b^{n}$

where $\varepsilon_{k r m n}:=[(k-r) w+(m-n) v]^{2}$. Inserting the power series (2.3) for the transformed Hamiltonian into the flow equation (2.1) and comparing the coefficients of the powers of $\lambda$ gives the differential equations:

$\frac{d H_{n}(\ell)}{d \ell}=\left[\left[H_{0}, H_{n}\right], H_{0}\right]+\sum_{\substack{a+b=n \\ a, b \neq 0}}\left[\left[H_{0}, H_{a}\right], H_{b}\right] \quad \forall n=0,1,2 \ldots$

It follows that $H_{0}=$ const. and $H_{k}(\ell), k=1,2 \ldots$ can be iteratively calculated: inserting the general ansatz

$H_{n}(\ell)=\sum_{k, r, m, n} \delta_{k r m n}(\ell) a^{\dagger k} a^{r} b^{\dagger m} b^{n}$

into (4.4) results in differential equations of the form

$\frac{d}{d \ell} \delta_{k r m n}(\ell)=-\varepsilon_{k r m n} \delta_{k r m n}(\ell)+\alpha_{k r m n}(\ell) \quad$ (no summation),

where $\varepsilon_{k r m n} \geq 0$ was defined above. The function $\alpha_{k r m n}(\ell)$ can be shown to be a sum of terms of the form

$c \ell^{n} \exp (-\gamma \ell) \quad(c=$ const., $\gamma>0, n=0,1,2 \ldots)$.

The solution to (4.6) is

$\delta_{k r m n}(\ell)=\exp \left(-\varepsilon_{k r m n} \cdot \ell\right) \int_{0}^{\ell} d \ell^{\prime} \alpha_{k r m n}\left(\ell^{\prime}\right) \exp \left(\varepsilon_{k r m n} \ell^{\prime}\right)$.

Ignoring for a moment the inhomogeneity $\alpha_{k r m n}(\ell)$ we find the following behavior: all terms will decay exponentially except for two cases in which $\varepsilon_{k r m n}$ may vanish: 
1. The given operator term $a^{\dagger k} a^{r} b^{\dagger m} b^{n}$ is diagonal $(k=r$ and $m=n)$.

2. The frequencies $w$ and $v$ are commensurate.

It can be seen that the inhomogeneity $\alpha_{k r m n}(\ell)$ will not change this general behavior. Thus the transformation can be successfully performed in the case of incommensurate frequencies and only diagonal terms will remain in the limit $\ell \rightarrow \infty$. In the case of commensurate frequencies off-diagonal operators $a^{\dagger k} a^{r} b^{\dagger m} b^{n}$ may only remain for $\varepsilon_{k r m n}=0$.

Since the number of terms in $H_{k}(\ell)$ grows rapidly with $k$ the described procedure with all its algebraic manipulations was performed by a computer program in the language $\mathrm{C}$. This way $H_{0}, \ldots, H_{8}$ were determined. The results of these calculations will be presented in the following section.

\section{Results of the Diagonalization}

The two procedures described in the last two sections were applied to the Hénon-Heiles Hamiltonian (3.1) in a case of incommensurate frequencies with the values $w=1.3, v=0.7, \lambda=-0.1, n=0.1$. The iteration procedure to 8 th order results in the following diagonal form (at $\lambda=-0.1$ ):

$$
\begin{aligned}
H= & 2.20910 \cdot 10^{-7} a^{\dagger 5} a^{5}+1.19855 \cdot 10^{-6} a^{\dagger 4} a^{4} b^{\dagger} b+2.17973 \cdot 10^{-6} a^{\dagger 4} a^{4} \\
& +1.54236 \cdot 10^{-6} a^{\dagger 3} a^{3} b^{\dagger 2} b^{2}+4.12440 \cdot 10^{-6} a^{\dagger 3} a^{3} b^{\dagger} b \\
& -8.65783 \cdot 10^{-5} a^{\dagger 3} a^{3}+6.09731 \cdot 10^{-8} a^{\dagger 2} a^{2} b^{\dagger 3} b^{3} \\
& -4.99568 \cdot 10^{-6} a^{\dagger 2} a^{2} b^{\dagger 2} b^{2}-0.00030 a^{\dagger 2} a^{2} b^{\dagger} b-0.00634 a^{\dagger 2} a^{2} \\
& -2.46444 \cdot 10^{-7} a^{\dagger} a b^{\dagger 4} b^{4}-6.97206 \cdot 10^{-6} a^{\dagger} a b^{\dagger 3} b^{3}-0.00022 a^{\dagger} a b^{\dagger 2} b^{2} \\
& -0.01121 a^{\dagger} a b^{\dagger} b+1.28264 a^{\dagger} a+3.44234 \cdot 10^{-9} b^{\dagger 5} b^{5} \\
& -2.84772 \cdot 10^{-7} b^{\dagger 4} b^{4}-1.59258 \cdot 10^{-5} b^{\dagger 3} b^{3}-0.00171 b^{\dagger 2} b^{2} \\
& +0.69148 b^{\dagger} b+0.99552
\end{aligned}
$$

Table 1 shows the eigenvalue spectra derived from the transformed Hamiltonian for the cut-off procedure carried out to fourth order and for the iteration procedure to fourth and to eighth order in $\lambda$. The numerical calculations were performed by diagonalizing a $900 \times 900$-matrix using FORTRAN-routines presented in [13]. The error $\Delta$ gives the relation of the deviation from the numerical value with respect to the total shift due to the coupling:

$\Delta=\left|\frac{E_{\text {Iter } 8}-E_{\text {num. }}}{E_{\text {num. }}-E_{\text {free }}}\right|$

It was calculated for the values of the iteration procedure to 8th order.

The calculated spectrum reproduces the numerical data well. For the fixed value of $\lambda=0.1$ the iteration procedure gives better results than the cut-off procedure. Iteration to higher orders does not necessarily improve the approximation of the numerical data: the ground state energy is more accurate for the iteration to 4th order than for the iteration to 8th order. Therefore there is no monotonous convergence of the determined eigenvalues to the exact ones with increasing order of iteration. Whether the eigenvalues determined in the flow equation procedure are always above the exact ones could not be shown analytically.

One can determine how the energy eigenvalues for a given pair of quantum numbers changes as the coupling strength $\lambda$ is increased. The result is shown in figure 2 for 12 eigenvalues in the cut-off procedure to 3rd order and the iteration procedure to 8th order. With growing coupling $\lambda$ the potential well in figure 1 becomes shallower and the eigenstates move closer together. The eigenvalues decrease as the coupling is increased. Moreover, one finds that states with higher energy at $\lambda=-0.1$ will drop faster 
Table 1: Comparison between flow equation calculations and numerical data in a case of incommensurate frequencies $(w=1.3, v=0.7, \lambda=-0.1, n=0.1)$

\begin{tabular}{|c|c|c|c|c|c|c|c|c|}
\hline $\mathrm{n}$ & $n_{1}$ & $n_{2}$ & Cut-off & Iterat. 4 & Iterat. 8 & Numerical & $E_{\text {free }}$ & $\Delta(\%)$ \\
\hline 1 & 0 & 0 & 0.995567 & 0.995521 & 0.995525 & 0.995519 & 1.00 & 0.133899 \\
2 & 0 & 1 & 1.687242 & 1.687013 & 1.687010 & 1.686994 & 1.70 & 0.123020 \\
3 & 1 & 0 & 2.278543 & 2.278179 & 2.278170 & 2.278132 & 2.30 & 0.173770 \\
4 & 0 & 2 & 2.375702 & 2.375106 & 2.375064 & 2.375036 & 2.40 & 0.112162 \\
5 & 1 & 1 & 2.959696 & 2.958536 & 2.958439 & 2.958353 & 3.00 & 0.206497 \\
6 & 0 & 3 & 3.060918 & 3.059734 & 3.059592 & 3.059551 & 3.10 & 0.101362 \\
7 & 2 & 0 & 3.549267 & 3.548183 & 3.548119 & 3.547947 & 3.60 & 0.330432 \\
8 & 1 & 2 & 3.637534 & 3.635141 & 3.634827 & 3.634664 & 3.70 & 0.249480 \\
9 & 0 & 4 & 3.742857 & 3.740832 & 3.740491 & 3.740435 & 3.80 & 0.094015 \\
10 & 2 & 1 & 4.219694 & 4.216860 & 4.216555 & 4.216180 & 4.30 & 0.447387 \\
11 & 1 & 3 & 4.312030 & 4.307926 & 4.307197 & 4.306912 & 4.40 & 0.306162 \\
12 & 0 & 5 & 4.421492 & 4.418334 & 4.417653 & 4.417578 & 4.50 & 0.090995 \\
$\vdots$ & $\vdots$ & $\vdots$ & $\vdots$ & $\vdots$ & $\vdots$ & $\vdots$ & $\vdots$ & $\vdots$ \\
80 & 1 & 14 & 11.502109 & 11.437765 & 11.386826 & 11.348431 & 12.10 & 5.108646 \\
81 & 8 & 1 & 11.496693 & 11.460618 & 11.465128 & 11.412886 & 12.10 & 7.603105 \\
82 & 7 & 3 & 11.535246 & 11.478808 & 11.477820 & 11.415802 & 12.20 & 7.908462 \\
83 & 6 & 5 & 11.591118 & 11.517846 & 11.506570 & 11.432484 & 12.30 & 8.540015 \\
84 & 5 & 7 & 11.664419 & 11.578700 & 11.552905 & 11.470273 & 12.40 & 8.887770 \\
85 & 0 & 16 & 11.659609 & 11.614802 & 11.583460 & 11.532429 & 12.20 & 7.644281 \\
\hline
\end{tabular}

than the lower states as the coupling is increased. This is due to the fact that the eigenstates to higher eigenvalues are more spread out in space such that the effect of the $\lambda \cdot q_{2}^{3}$-term in the potential (3.1) upon them is larger.

Comparison with numerical data seems to indicate that for larger coupling values of $\lambda$ the cut-off procedure gives more accurate results than the iteration procedure. However, a precise quantitative analysis is not possible in the $\lambda$-range in which the eigenvalues from the two flow equation procedures differ: for growing values of $\lambda$ one has to restrict the numerical diagonalization to smaller matrices in order to avoid the effect of the continuum causing the appearance of intermittent states (seen in figure B).

\section{A Case of Commensurate Frequencies}

The two flow equation procedures (sections 3 and 4 ) were applied to the Hamiltonian (3.1) in a case of commensurate frequencies $(w=1.0, v=1.0, \lambda=-0.1, n=0.1)$. As shown in section $\sharp$, off-diagonal terms will not entirely disappear from the transformed Hamiltonian due to the commensurability. According to (4.3) and (4.6) remaining off-diagonal operator terms are of the form $a^{\dagger k} a^{r} b^{\dagger m} b^{n}$ with $\varepsilon_{k r m n}=(k-r)+$ $(m-n)=0$. They couple states $\left|n_{1}, n_{2}\right\rangle$ for which $n_{1}+n_{2}=$ const. Neglecting these off-diagonal terms, we obtained fairly accurate eigenvalues. To account for the off-diagonal terms small tridiagonal matrices were numerically diagonalized within the originally degenerate subspace. This improves the precision of the calculated eigenvalues. The cut-off procedure does not have this problem. Since the oscillator frequencies $v$ and $w$ in (3.3) are $\ell$ dependent (see (3.7)), initially commensurate frequencies become incommensurate for finite $\ell$. The results for the cut-off procedure to fourth order, iteration procedures to 

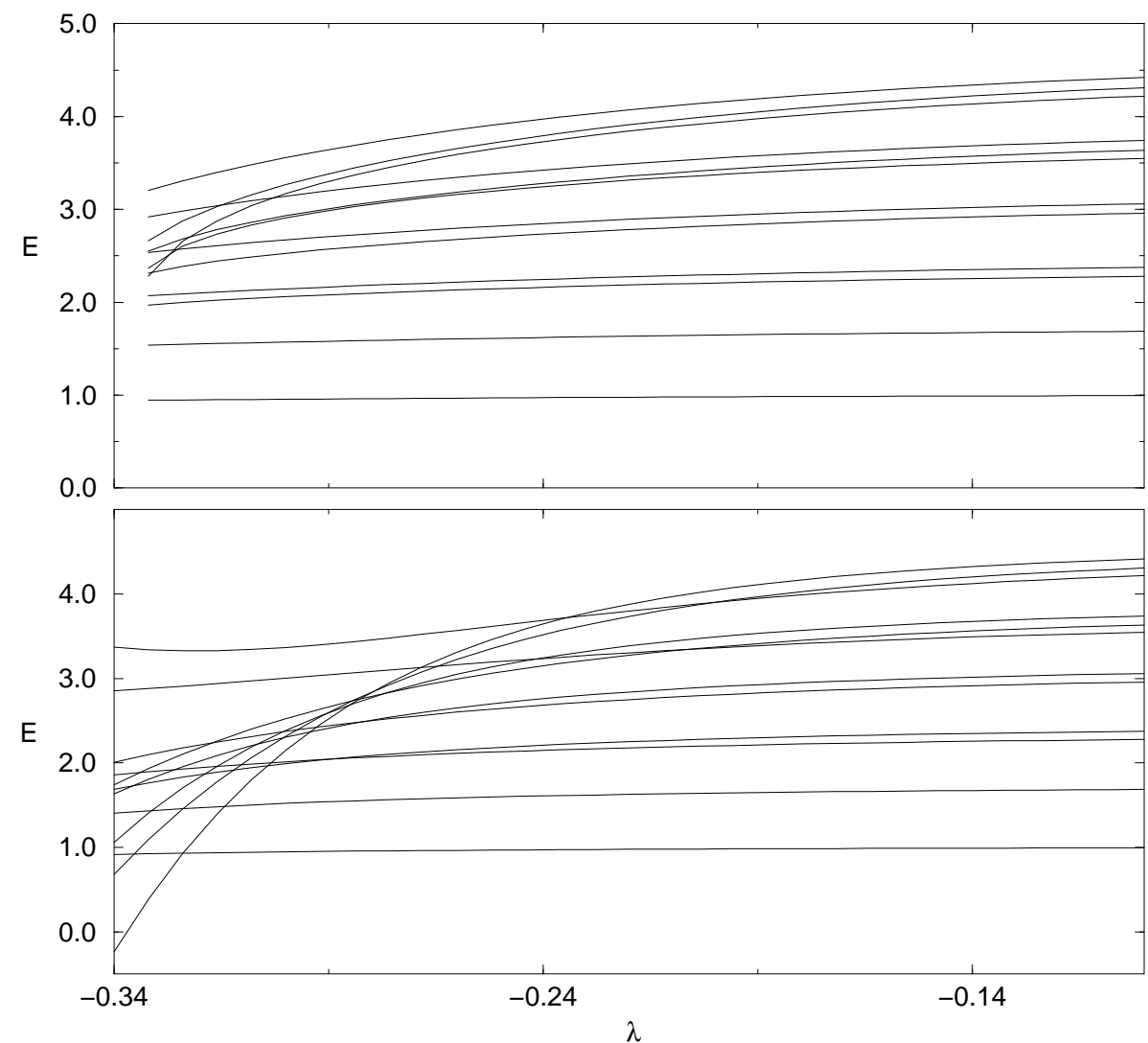

Figure 2: The eigenvalues for the quantum numbers of the lowest 12 eigenstates at $\lambda=-0.1$ as a function of the coupling strength $\lambda$ in the Cut-off procedure to 3rd order (above) and the iteration procedure to 8th order (below). The numerical integration breaks down for large coupling (above).

4th and 6th order and the improved values of the 6th-order iteration are listed in table $2 . \Delta$ gives the error of the improved values with respect to the total shift due to the coupling:

$\Delta=\left|\frac{E_{\text {impr. }}-E_{\text {num. }}}{E_{\text {num. }}-E_{\text {free }}}\right|$

The originally degenerate subspaces are separated by horizontal lines.

\section{Dynamics of the Quantum Mechanical System}

In the framework of classical mechanics one can put a particle in the potential well (figure 1) and calculate the trajectory for a given set of starting values. To model this classical approach in the framework of quantum mechanics one can ask: How does a state originally located within the potential well evolve with time? The eigenstates of the uncoupled oscillator are located within the potential well. Their time evolution is given by the Hamilton operator $H$ of the coupled system. The absolute value of the matrix element

$\langle\beta|\exp (i H t)| \alpha\rangle$ 


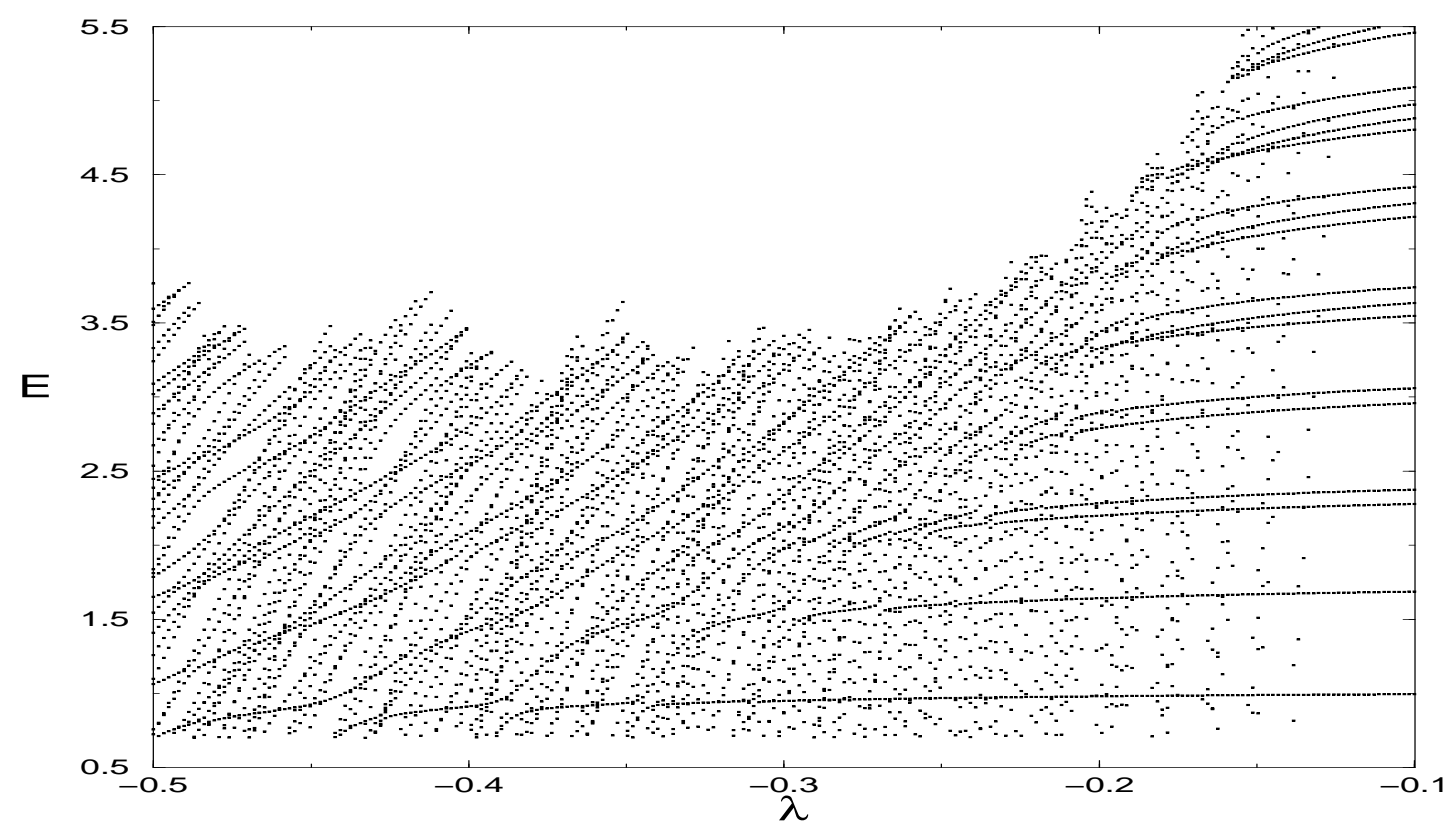

Figure 3: Numerical diagonalization of a $900 \times 900-$ matrix. For 256 $\lambda$-values in the interval $[-0.5,-0.1]$ the first 29 eigenvalues $\mathrm{E}$ above 0.7 were determined. With growing perturbation the effect of the continuum becomes dominant.

indicates how much of a particle is in state $|\beta\rangle$ after time $t$ if the particle was located in state $|\alpha\rangle$ at time 0 where $|\alpha\rangle$ and $|\beta\rangle$ represent two of the eigenstates of the uncoupled oscillator.

To calculate such matrix elements the eigenstates of the uncoupled oscillator are expressed in terms of the eigenstates of the full Hamiltonian of the coupled system. For this purpose one can set up flow equations for the transformation of states which results in a fairly large set of differential equations. Since the transformation of the Hamiltonian was already calculated there is an easier way to calculate the transformation of states which will be explained in the framework of the iteration procedure introduced in section 4 :

In analogy to the transformation of the Hamiltonian one determines the transformed annihilation operator $a(\ell)=U^{\dagger}(\ell) a U(\ell)$ from the flow equations

$\frac{d a(\ell)}{d \ell}=[\eta(\ell), a(\ell)]$,

and the generator $\eta$ already known from the calculation of $H(\ell)$. One obtains the annihilation operator as a power series in the coupling constant $\lambda$ similar to the one obtained for the transformed Hamiltonian (2.3, 4.5). By definition the transformed ground state of the uncoupled oscillator $|0\rangle$ is given by:

$a(\ell=\infty)|0\rangle=0 \quad, \quad b(\ell=\infty)|0\rangle=0 \quad$ and $\quad\langle 0 \mid 0\rangle=1$.

These equations can be solved for $|0\rangle$ as a function of the eigenstates $|n, m\rangle_{\infty}$ of the full Hamiltonian:

$|0\rangle=\sum_{n, m, i=0}^{\infty} c_{n m}^{(i)} \lambda^{i}|n, m\rangle_{\infty}$.

One can then construct any exited state $|\alpha\rangle=a^{\dagger k}(\infty) b^{\dagger m}(\infty)|0\rangle$. In the following the abbreviations $a:=a(\infty)$ and $b:=b(\infty)$ will be used. 
Table 2: Comparison between several flow equation calculations and numerical data in a case of commensurate frequencies

$(w=1.0, v=1.0, \lambda=-0.1, n=0.1)$

\begin{tabular}{|c|c|c|c|c|c|c|c|c|c|}
\hline $\mathrm{n}$ & $n_{1}$ & $n_{2}$ & Cut-off & Iterat. 4 & Iterat. 6 & improved & Numerical & $E_{\text {free }}$ & $\Delta(\%)$ \\
\hline 1 & 0 & 0 & 0.997021 & 0.996989 & 0.996990 & 0.996990 & 0.996987 & 1 & 0.084 \\
\hline 2 & 1 & 0 & 1.983815 & 1.983444 & 1.983415 & 1.983415 & 1.983420 & 2 & 0.030 \\
3 & 0 & 1 & 1.991400 & 1.991187 & 1.991180 & 1.991180 & 1.991170 & 2 & 0.111 \\
\hline 4 & 2 & 0 & 2.962272 & 2.961007 & 2.960816 & 2.956997 & 2.957081 & 3 & 0.195 \\
5 & 1 & 1 & 2.968458 & 2.967112 & 2.966985 & 2.966985 & 2.966957 & 3 & 0.084 \\
6 & 0 & 2 & 2.985028 & 2.984515 & 2.984469 & 2.988288 & 2.988269 & 3 & 0.162 \\
\hline 7 & 3 & 0 & 3.932391 & 3.929514 & 3.928861 & 3.917954 & 3.918277 & 4 & 0.395 \\
8 & 2 & 1 & 3.937177 & 3.933515 & 3.933009 & 3.926540 & 3.926527 & 4 & 0.018 \\
9 & 1 & 2 & 3.952348 & 3.949675 & 3.949312 & 3.960219 & 3.960177 & 4 & 0.105 \\
10 & 0 & 3 & 3.977903 & 3.976969 & 3.976841 & 3.983311 & 3.983255 & 4 & 0.331 \\
\hline 11 & 4 & 0 & 4.894173 & 4.888802 & 4.887144 & 4.865409 & 4.865039 & 5 & 0.274 \\
12 & 3 & 1 & 4.897560 & 4.890232 & 4.888878 & 4.870960 & 4.871053 & 5 & 0.072 \\
13 & 2 & 2 & 4.911331 & 4.904683 & 4.903555 & 4.914437 & 4.916403 & 5 & 2.352 \\
14 & 1 & 3 & 4.935486 & 4.931130 & 4.930349 & 4.948267 & 4.948041 & 5 & 0.435 \\
15 & 0 & 4 & 4.970025 & 4.968546 & 4.968277 & 4.979130 & 4.978266 & 5 & 3.973 \\
\hline 16 & 4 & 1 & 5.849605 & 5.837100 & 5.834141 & 5.796496 & 5.795370 & 6 & 0.550 \\
17 & 5 & 0 & 5.847617 & 5.838708 & 5.835184 & 5.799784 & 5.799166 & 6 & 0.308 \\
$\vdots$ & $\vdots$ & $\vdots$ & $\vdots$ & $\vdots$ & $\vdots$ & $\vdots$ & $\vdots$ & $\vdots$ & $\vdots$ \\
\hline
\end{tabular}

For several final states $|\beta\rangle$ matrix elements of the type (7.1) are determined as a function time. Because of the coupling of time and energy in the exponential function an expansion of the exponent in powers of $\lambda$ - and thus in powers of $t$ - does not give the right long term behavior. Therefore the exponential functions are not expanded. The resulting matrix elements are of the form:

$\langle\beta|\exp (i H t)| \alpha\rangle=\sum_{k} a_{k} \lambda^{b_{k}} e^{i E_{k} t}$,

with coefficients $a_{k}$ and exponents $b_{k}$. $E_{k}$ represents a difference of energy eigenvalues which are determined in the iterative process to the 6th power of $\lambda$. Thus if the coefficients of the expansion in $\lambda$ are all of order 1 the phases $E_{k} t$ can be determined up to 1 percent accuracy in the range

$t<t_{\max } \approx \frac{0.01}{\lambda^{7}}=10^{5}$.

This gives only a rough estimate since Siegel [14] proved that the Birkhoff normal form will generally not converge. This can easily be deduced from the fact that the Hénon-Heiles potential is not integrable whereas any polynomial in Birkhoff normal form will always be integrable. One expects an asymptotic convergence of the expansion in $\lambda$ such that the coefficients of higher powers of $\lambda$ will generally grow. Thus the time range in which the calculated matrix elements are valid is smaller than the one given above.

For the initial state $|\alpha\rangle=a^{\dagger}|0\rangle$ amplitudes for the transition to the six final states $|\beta\rangle=a^{\dagger}|0\rangle, a^{\dagger} b^{\dagger}|0\rangle$, $a^{\dagger} b^{\dagger 2}|0\rangle, a^{\dagger 3}|0\rangle, a^{\dagger 3} b^{\dagger}|0\rangle$ and $a^{\dagger} b^{\dagger 3}|0\rangle$ were determined at $\lambda=-0.1$. Figure $\emptyset$ shows the first four of them as a function of time. Amplitudes for transitions to final states with an even number of $a^{\dagger}$-operators can be shown to vanish. 


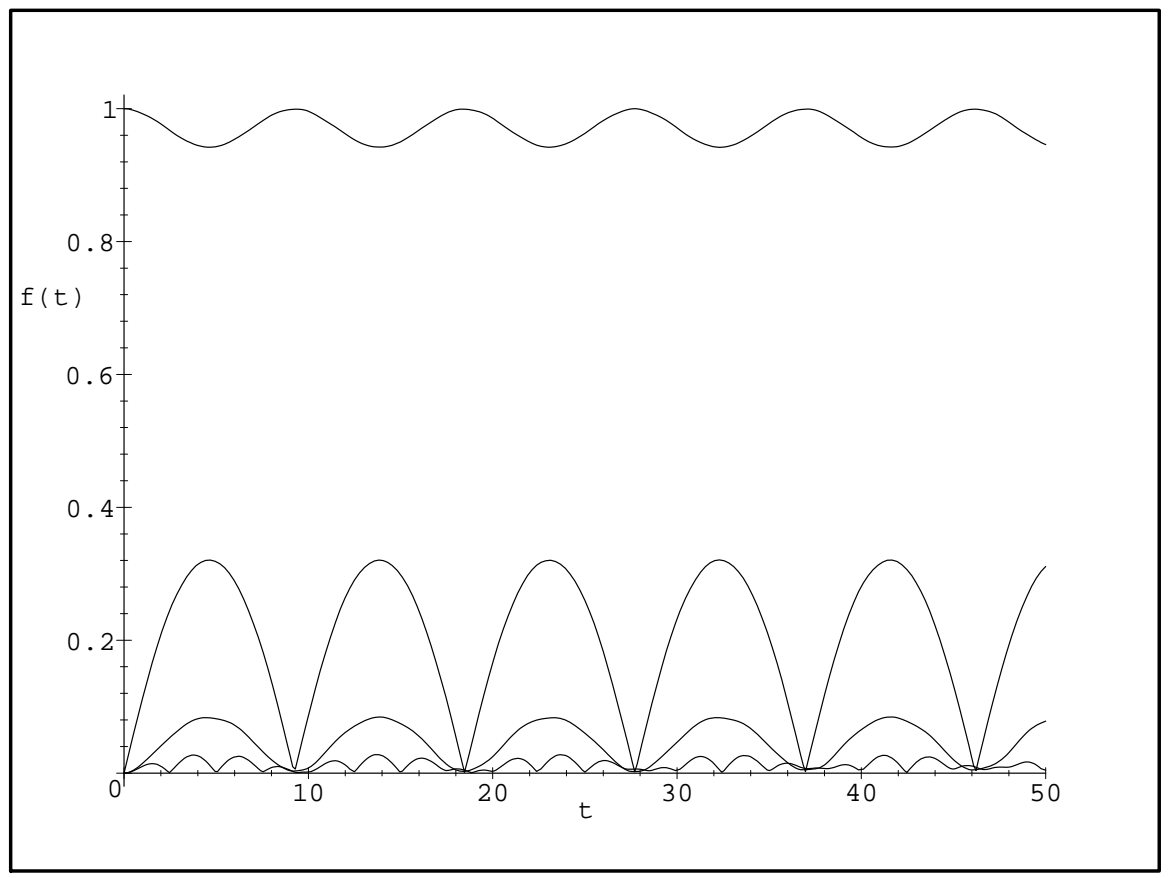

Figure 4: Transition amplitudes $f(t)=\left\langle 0\left|a U a^{\dagger}\right| 0\right\rangle,\left\langle 0\left|a b U a^{\dagger}\right| 0\right\rangle$, $\left\langle 0\left|a b^{2} U a^{\dagger}\right| 0\right\rangle$ and $\left\langle 0\left|a^{3} U a^{\dagger}\right| 0\right\rangle$ (in order of size) where $U:=\exp (i H t), a=a(\infty)$ and $b=b(\infty)$

The sum of their absolute values was subtracted from 1 - in figure 5 - to show that these are indeed the relevant transitions. The amount of negative value in this figure gives an indication of the numerical error.

The parameter $\lambda$ is a measure of the strength of coupling between the two harmonic oscillators (3.1). One expects that a growing coupling value facilitates transitions between different states. Figure 6 shows the square of the transition amplitude $\left\langle 0\left|a \exp (i H t) a^{\dagger}\right| 0\right\rangle$ as a function of time for various values of $\lambda$. Moreover, one finds that the dominant oscillation frequency decreases with growing coupling.

In the framework of quantum mechanics one expects a particle located within the potential well of figure 1 to tunnel through the potential barrier with a certain probability. This probability depends upon the energy of the particle and upon the size and geometry of the barrier. Thus the tunneling should become apparent if one investigates the dynamics of higher exited states or if one decreases the size of the potential well by increasing the coupling $\lambda$. Estimates of the tunneling frequency show that the effect of tunneling is negligible for $\lambda=-0.1$. However, for $\lambda=-0.25$ tunneling should become apparent. In figure 6 one finds a modulation in the amplitude of oscillation for $\lambda=-0.25$. But this may also be a numerical artifact because in this range of coupling strength the numerical precision decreases. Since the Birkhoff approach consists in approximating a power series by a finite polynomial, any transition amplitude will always be a finite sum of harmonic oscillations. Therefore any calculated time evolution will necessarily be periodic in time.

Physical observables can be calculated in essentially the same way as was shown for the transition amplitudes (7.1). Using the relations

$\hat{q}=\frac{1}{\sqrt{2}}\left(a^{\dagger}+a\right), \quad \hat{p}=\frac{i}{\sqrt{2}}\left(a^{\dagger}-a\right)$. 


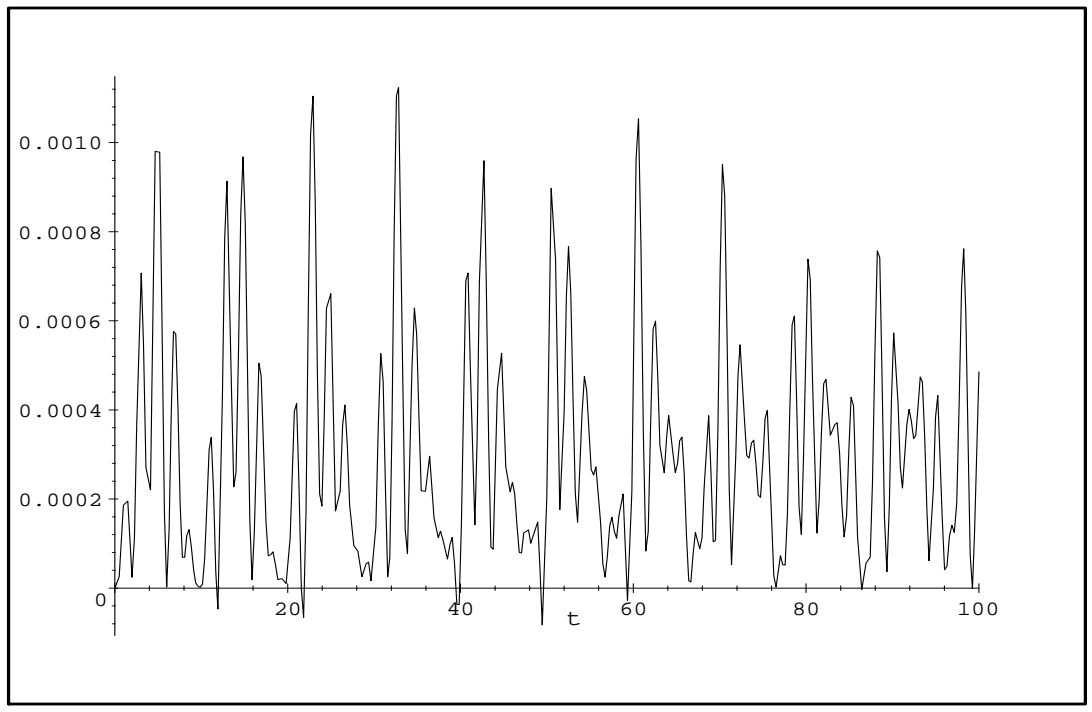

Figure 5: $\left(1-\sum_{\beta}|\langle\beta|U| \alpha\rangle|^{2}\right)$ with initial state $|\alpha\rangle=a^{\dagger}|0\rangle$ and the six final states $|\beta\rangle$ mentioned above $(U:=\exp (i H t))$

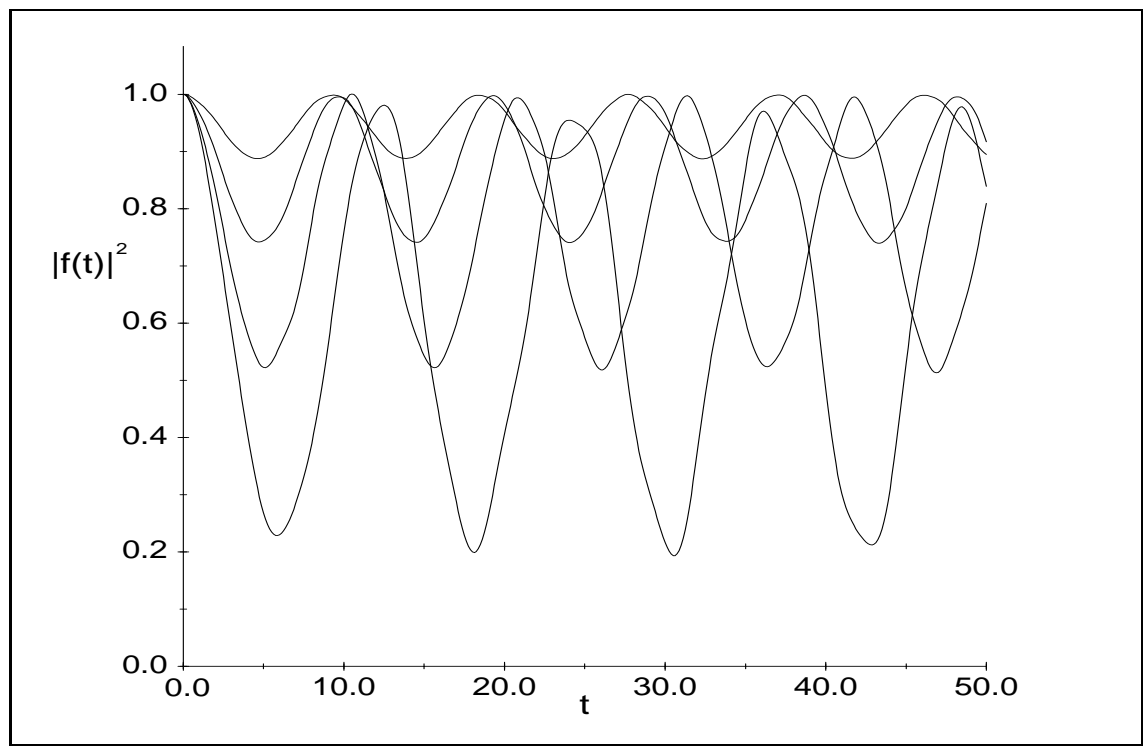

Figure 6: Evolution of the first exited state $|f(t)|^{2}$ for the coupling values $\lambda=-0.1,-0.15,-0.2$ and -0.25 (in the order of growing amplitude), where $f(t)=$ $\left\langle 0\left|a \exp (i H t) a^{\dagger}\right| 0\right\rangle$.

for spatial and momentum coordinates one can easily calculate expectation values of the form $\left\langle\hat{q}^{2}\right\rangle=$ $\left\langle\alpha\left|U^{\dagger} \hat{q}^{2} U\right| \alpha\right\rangle$, which show a similar oscillatory behavior as that in figure $\emptyset$. 


\section{Summary and Outlook}

In this paper we calculated effective eigenvalues of the quantum mechanical Hénon-Heiles Hamiltonian using flow equations - a method of continuous unitary transformation proposed by Wegner [细]. We used two different procedures to solve the flow equations - an iterative procedure and a Cut-off procedure. The Cut-off procedure has been used before in several applications of the flow equations. It seems to be most appropriate if a (perturbative) renormalization of the Hamiltonian has to be done. This is not necessary in the present case. The Cut-off procedure has the disadvantage that in most cases it is difficult to solve the resulting differential equations explicitly. Often one can extract the asymptotic behavior and based thereon an approximate solution. But if one wants to have precise numerical results, one has to solve the differential equations numerically. The main advantage of the iterative procedure is that the differential equations can be solved explicitly and that it can be carried out to much higher orders.

We used both methods to calculate the eigenvalue spectrum of the Hénon-Heiles Hamiltonian. In a case of incommensurate frequencies the eigenvalues coincide well with those obtained by numerical diagonalization of a finite matrix. In the treated case the precision of results is comparable to that obtained by Delos and Swimm [7] who used consecutive canonical transformations to approximate the classical Hamilton function by a Birkhoff normal form. For small coupling value the iteration procedure gives more accurate results whereas for larger coupling values the cut-off procedure seems to be better. However, for very large coupling the potential well decreases in size and the discrete spectrum disappears. This is found in a series of numerical matrix diagonalizations at various coupling values.

The case of commensurate frequencies can be treated in essentially the same way, matrix diagonalization shows good agreement with numerical results. In the commensurate case the diagonalization procedure is much less lengthy than that presented by Delos and Swimm. A quantitative comparison to their results is not possible since the normal form cited in [7] does not correspond to the given parameter set. And the presented eigenvalue spectrum does not match with either the parameter set given or the normal form cited.

On the basis of the transformation of eigenstates we analyzed the quantum mechanical dynamics of the Hénon-Heiles system. We determined the time evolution of a set of states located within the potential well. We found oscillations between different states with amplitudes depending on the coupling strength. A certain completeness is found by adding up absolute values of several transition amplitudes.

It is interesting to see that continuous unitary transformations can be used to obtain precise results for a system like the quantum mechanical Hénon-Heiles model. The method was originally designed to calculate eigenvalues of a given Hamiltonian, at least approximatively and close to the ground state [2]. In many applications of the method, the goal was to obtain an effective Hamiltonian that describes well the low energy behavior of the system (see e.g. [8, 9, 10, 11]). The effective Hamiltonian calculated in the present approach is in a quantum mechanical Birkhoff normal form, i.e. a polynomial in the oscillator Hamiltonians $\left(p^{2}+q^{2}\right)$. It does not describe the $q^{3}$-shape of the Hénon-Heiles potential for large absolute values of $q$. The energies we calculated using this method are energies of approximately stationary states. As a consequence one cannot describe the tunneling through the barrier. This limitation is not imposed by the flow equation method. Even numerical diagonalizations will not allow a correct description of the tunneling. But for sufficiently large escape times the results describe the short time behavior of the system quite well.

The transformation to a Birkhoff normal form yields an asymptotic series because the classical Hénon-Heiles system shows a transition to a chaotic regime. In principle the flow equations can be successfully applied to a quantum mechanical Hamiltonian that has a chaotic classical counterpart (see also [12]). A first focus of interest for such a system are statistical properties of the spectrum [3]. Flow equations can be used to calculate precise eigenenergies. The way normal ordering is introduced determines the interval of energy where precision is high. Typically one has to restrict the number of couplings 
in the Hamiltonian using some truncation scheme. The neglected terms are in a normal ordered form. If one chooses the ground state as the basis for the normal ordering, the resulting effective Hamiltonian describes the ground state and low lying excitations quite well. In the present approach the normal ordering was introduced with respect to the ground state of the uncoupled system. Therefore we were able to obtain precise results for energies close to the minimum of the potential. One could also choose a normal ordering with respect to some high energy state in order to calculate eigenenergies close to this energy scale. While this approach is not sensible in the case of the Hénon-Heiles system, because the potential well has only a limited depth, it can be useful for other physical systems.

\section{References}

[1] M. Hénon, C. Heiles: Astron. J. 69, 73 (1964)

[2] F. Wegner: Ann. Physik (Leipzig) 3, 77 (1994)

[3] M. C. Gutzwiller: Chaos in Classical and Quantum Mechanics. New York: Springer-Verlag (1990)

[4] F. G. Gustavson: Astron. J. 71, 670 (1966)

[5] G. D. Birkhoff: Dynamical Systems. New York: American Mathematical Society Colloquium Publications Vol. IX (1927)

[6] J. B. Delos and R. T. Swimm: Chem. Phys. Lett. 47, 76 (1977)

[7] J. B. Delos and R. T. Swimm: J. Chem. Phys. 71, 1706 (1979)

[8] S. K. Kehrein and A. Mielke: Phys. Lett. A 219, 313 (1996)

[9] S. K. Kehrein and A. Mielke: Ann. Physik (Leipzig) 6, 90 (1997)

[10] P. Lenz and F. Wegner: Nucl. Phys. B482 [FS], 693 (1996)

[11] A. Mielke: Europhys. Lett. 40, 195-200 (1997)

[12] A. Mielke: 'Flow equations for band-matrices.' Preprint HD-TVP-98-01, to appear in European Physical Journal B (1998).

[13] B. T. Smith, J. M. Boyle, J. J. Dongarra, B. S. Garbow, Y. Ibeke, V. C. Klema and C. B. Moler: Matrix Eigensystem Routines-EISPACK Guide, Lecture Notes in Computer Science, Volume 62nd Edition, G. Goos and J. Hartmanis, Springer-Verlag, New York, Heidelberg, Berlin (1976)

[14] C. L. Siegel: Ann Math 42, 806 (1941) and 128, 144 (1945) 